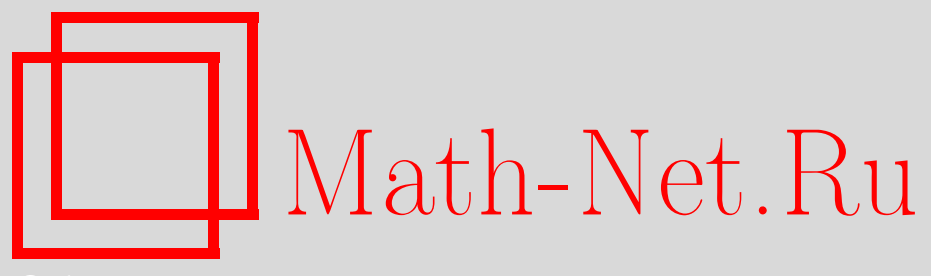

Б. А. Дубровин, Гамильтоновы уравнения в частных производных и фробениусовы многообразия, УМН, 2008, том 63, выпуск 6, 7-18

DOI: https://doi.org/10.4213/rm9242

Использование Общероссийского математического портала Math-Net.Ru подразумевает, что вы прочитали и согласны с пользовательским соглашением http://www.mathnet.ru/rus/agreement

Параметры загрузки:

IP: 54.157 .27 .8

26 апреля 2023 г., 14:50:18

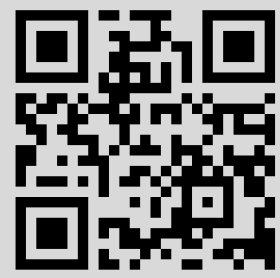




\section{Гамильтоновы уравнения в частных производных и фробениусовы многообразия}

\section{Б. А. Дубровин}

Первая часть работы посвящена приложениям теории фробениусовых многообразий к задаче классификации гамильтоновых систем уравнений в частных производных, зависящих от малого параметра. Попутно развивается теория деформаций интегрируемых иерархий, в том числе так называемых иерархий топологического типа. K их числу относятся как хорошо известные иерархии, такие как иерархия уравнения Кортевега-де Фриза, нелинейного уравнения Шрёдингера, Тоды, Буссинеска и т.д., так и ряд новых иерархий, некоторые из которых могут играть важную роль в приложениях. Во второй части работы мы изучаем свойства решений этих уравнений, уделяя особое внимание сопоставлению свойств решений возмущенных и невозмущенных уравнений в окрестности точки градиентной катастрофы. Формулируется гипотеза универсалъности, описывающая различные типы критического поведения решений возмущенной системы в окрестности точки градиентной катастрофы невозмущенной системы.

Библиография: 10 названий.

Для данного $n$-мерного многообразия $M^{n}$ обозначим через

$$
\mathscr{L}\left(M^{n}\right)=\left\{S^{1} \rightarrow M^{n}\right\}
$$

пространство петель со значениями в $M^{n}$. Основным объектом нашего исследования являются гамильтоновы векторные поля, зависящие от малого параметра $\varepsilon$. Для краткости обозначений мы их будем называть векторными полями на расширенном пространстве петель $\mathscr{L}\left(M^{n}\right) \otimes \mathbb{R}[[\varepsilon]]$. Более точно, будут изучаться зависящие от параметра $\varepsilon$ эволюционные системы уравнений в частных производных с одной пространственной переменной $x$, записываемые в виде

$$
u_{t}^{i}=A_{j}^{i}(u) u_{x}^{j}+\varepsilon\left(B_{j}^{i}(u) u_{x x}^{j}+\frac{1}{2} C_{j k}^{i}(u) u_{x}^{j} u_{x}^{k}\right)+O\left(\varepsilon^{2}\right), \quad i=1, \ldots, n .
$$

Здесь $u=\left(u^{1}, \ldots, u^{n}\right)$ - локальные координаты на $M^{n}$. Топология этого многообразия будет предполагаться тривиальной ( $n$-мерный шар), хотя мы и будем делать нелинейные замены координат в этом шаре.

В разложении правых частей системы (1) предполагается, что члены порядка $\varepsilon^{k}$ являются полиномами степени $k+1$ по производным $u_{x}, \ldots, u^{(k+1)}$, где, по определению,

$$
\operatorname{deg} u^{(m)}=m, \quad m=1,2, \ldots .
$$

(C) Б. А. Дувровин, 2008 
Будет предполагаться, что система (1) является гамильтоновой по отношению к локальным скобкам Пуассона

$$
\begin{gathered}
u_{t}^{i}=\left\{u^{i}(x), H\right\}=\sum_{k \geqslant 0} \varepsilon^{k} \sum_{m=0}^{k+1} A_{k, m}^{i j}\left(u ; u_{x}, \ldots, u^{(m)}\right) \partial_{x}^{k-m+1} \frac{\delta H}{\delta u^{j}(x)}, \\
\left\{u^{i}(x), u^{j}(y)\right\}=\sum_{k \geqslant 0} \varepsilon^{k} \sum_{m=0}^{k+1} A_{k, m}^{i j}\left(u(x) ; u_{x}(x), \ldots, u^{(m)}(x)\right) \delta^{(k-m+1)}(x-y), \\
\quad \operatorname{deg} A_{k, m}^{i j}\left(u ; u_{x}, \ldots, u^{(m)}\right)=m,
\end{gathered}
$$

с локальным гамильтонианом

$$
\begin{gathered}
H=\sum_{k \geqslant 0} \varepsilon^{k} \int h_{k}\left(u ; u_{x}, \ldots, u^{(k)}\right) d x, \\
\operatorname{deg} h_{k}\left(u ; u_{x}, \ldots, u^{(k)}\right)=k .
\end{gathered}
$$

В формуле $(3) \delta(x)$ - это дельта-функция Дирака. Смысл этих обозначений ясен из явного выражения (2). Интеграл в (4) понимается в смысле формального вариационного исчисления. Другими словами, интеграл дифференциального полинома $h=h\left(u ; u_{x}, \ldots, u^{(m)}\right)$ определяется как класс эквивалентности этого полинома по модулю полных производных:

$$
\begin{gathered}
h\left(u ; u_{x}, \ldots, u^{(m)}\right) \sim h\left(u ; u_{x}, \ldots, u^{(m)}\right)+\partial_{x}\left(f\left(u ; u_{x}, \ldots, u^{(m-1)}\right)\right), \\
\partial_{x}=\sum_{k \geqslant 0} u^{i^{(k+1)}} \frac{\partial}{\partial u^{i(k)}}, \quad \text { где } u^{i^{(k)}}:=\frac{d^{k} u^{i}}{d x^{k}} .
\end{gathered}
$$

Далее, $\delta H / \delta u^{j}(x)$ - это оператор Эйлера-Лагранжа:

$$
\frac{\delta H}{\delta u^{j}(x)}=\frac{\partial h}{\partial u^{j}}-\partial_{x} \frac{\partial h}{\partial u_{x}^{j}}+\partial_{x}^{2} \frac{\partial h}{\partial u_{x x}^{j}}-\cdots \quad \text { для } H=\int h d x .
$$

Коэффициенты скобок Пуассона и плотностей гамильтониана предполагаются полиномиальными по производным в каждом порядке по $\varepsilon$. Антисимметрия и тождество Якоби должны выполняться как соотношения для формальных

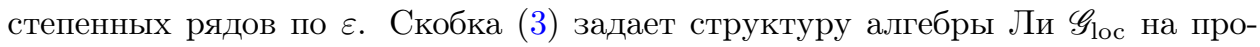
странстве всех локальных функционалов:

$$
\begin{gathered}
\{F, G\}=\int \frac{\delta F}{\delta u^{i}(x)} A^{i j} \frac{\delta G}{\delta u^{j}(x)} d x \\
A^{i j}:=\sum_{k \geqslant 0} \varepsilon^{k} \sum_{m=0}^{k+1} A_{k, m}^{i j}\left(u ; u_{x}, \ldots, u^{(m)}\right) \partial_{x}^{k-m+1}, \\
F=\sum_{k \geqslant 0} \varepsilon^{k} \int f_{k}\left(u ; u_{x}, \ldots, u^{(k)}\right) d x, \quad G=\sum_{l \geqslant 0} \varepsilon^{l} \int g_{l}\left(u ; u_{x}, \ldots, u^{(l)}\right) d x \\
\operatorname{deg} f_{k}\left(u ; u_{x}, \ldots, u^{(k)}\right)=k, \quad \operatorname{deg} g_{l}\left(u ; u_{x}, \ldots, u^{(l)}\right)=l .
\end{gathered}
$$

Полное кольцо функций на бесконечномерном "многообразии" $\mathscr{L}\left(M^{n}\right) \otimes \mathbb{C}[[\varepsilon]]$ строится как подходящим образом пополненная симметрическая тензорная алгебра над $\mathscr{G}_{\text {loc. }}$ 
Предыдущие формулы определяют класс функций, векторных полей и скобок Пуассона на бесконечномерном "многообразии" $\mathscr{L}\left(M^{n}\right) \otimes \mathbb{C}[[\varepsilon]]$. Чтобы развивать геометрический подход к изучению этих объектов, следует ввести класс допустимых “замен координат" на этом "многообразии". Таковые замены вводятся через так называемые обобщенные преобразования Миуры

$$
\begin{gathered}
u^{i} \mapsto \tilde{u}^{i}=\sum_{k \geqslant 0} \varepsilon^{k} F_{k}^{i}\left(u ; u_{x}, \ldots, u^{(k)}\right), \\
\operatorname{deg} F_{k}^{i}\left(u ; u_{x}, \ldots, u^{(k)}\right)=k, \quad \operatorname{det}\left(\frac{\partial F_{0}^{i}(u)}{\partial u^{j}}\right) \neq 0 .
\end{gathered}
$$

Коэффициенты $F_{k}^{i}\left(u ; u_{x}, \ldots, u^{(k)}\right)$ должны быть дифференциальными полиномами. Легко видеть, что преобразования вида (6) образуют группу. В самом деле, для того чтобы обратить преобразование (6), нужно решить дифференциальное уравнение для $u^{1}, \ldots, u^{n}$. Необходимое решение получается в виде ВКБ-разложения по малому параметру. Несложное вычисление показывает, что класс эволюционных уравнений в частных производных (1), скобок Пуассона (3), а также класс локальных гамильтонианов (4) инвариантен по отношению к группе обобщенных преобразований Миуры.

Мы скажем, что два объекта нашей теории (т. е. две эволюционные системы вида (1), две локальные скобки Пуассона (3) или же два локальных гамильтониана (4)) эквивалентны, если один из них получается из другого посредством обобщенного преобразования Миуры.

Основными задачами нашего исследования являются:

- классификация общих гамильтоновых систем,

- применение к изучению интегрируемых систем,

- выработка новых подходов к изучению свойств решений.

Начнем с классификации скобок Пуассона.

Теорема 1. В предположении

$$
\operatorname{det}\left(A_{0,0}^{i j}(u)\right) \neq 0
$$

любая скобка вида (3) эквивалентна скобке следующего стандартного вида:

$$
\left\{\tilde{u}^{i}(x), \tilde{u}^{j}(y)\right\}=\eta^{i j} \delta^{\prime}(x-y), \quad \eta^{i j}=\eta^{j i}=\text { const }, \quad \operatorname{det}\left(\eta^{i j}\right) \neq 0 .
$$

В доказательстве этой теоремы используется теория скобок Пуассона гидродинамического типа, развитая С. П. Новиковым и автором в 1983 г. Согласно этой теории, главный член скобки Пуассона задает (контравариантную) метрику нулевой кривизны

$$
g^{i j}(u):=A_{0,0}^{i j}(u)
$$

на многообразии $M^{n}$. Также используется тривиальность пуассоновых когомологий скобки (8), доказанная в 2001 г. Э. Гетцлером.

Следующим шагом в реализации нашей программы исследований является классификация бигамильтоновых структур вида (3), (7). Напомним, что системы

$$
u_{t}^{i}=\left\{u^{i}(x), H_{1}\right\}_{1}=\left\{u^{i}(x), H_{2}\right\}_{2}, \quad i=1, \ldots, n,
$$


являющиеся гамильтоновыми по отношению к двум совместным скобкам Пуассона указанного вида $\{\cdot, \cdot\}_{1}$ и $\{\cdot, \cdot\}_{2}$, всегда могут быть включены в максимальную абелеву подалгебру гамильтоновых векторных полей (интегрируемую иерархию), что влечет интегрируемость (это можно доказать).

ТЕОРема 2. При выполнении дополнителъных условий сильной невырожденности и полупростоты каждая бигамильтонова структура однозначно определяется:

1) пучком скобок Пуассона гидродинамического типа

$$
\begin{aligned}
\left\{v^{i}(x), v^{j}(y)\right\}_{2}-\lambda\left\{v^{i}(x), v^{j}(y)\right\}_{1}= & \left(g_{2}^{i j}(v(x))-\lambda g_{1}^{i j}(v(x))\right) \delta^{\prime}(x-y) \\
& +\left(\Gamma_{k 1}^{i j}(v)-\lambda \Gamma_{k 2}^{i j}(v)\right) v_{x}^{k} \delta(x-y)
\end{aligned}
$$

2) набором из $n$ функций одной переменной

$$
c_{1}\left(w^{1}\right), \ldots, c_{n}\left(w^{n}\right),
$$

называемых иентральными инвариантами.

Доказательство этой теоремы основано на

- теореме квазитривиальности: любая бигамильтонова структура делается эквивалентной бездисперсной (9), если расширить класс преобразований (6), допуская преобразования, рационально зависящие от производных;

- вычислении бигамильтоновых когомологий, т. е. деформаций пары антикоммутирующих ацикличных дифференциалов, задаваемых бигамильтоновой структурой.

Центральные инварианты, параметризующие инфинитезимальные деформации, строятся так. Каждой скобке Пуассона (3) поставим в соответствие ряд из матриц, зависящих от вспомогательной переменной $p$ :

$$
\pi^{i j}(u ; p)=\sum_{k \geqslant 0} A_{k, 0}^{i j}(u) p^{k} .
$$

Напомним, что степень коэффициентов $A_{k, 0}^{i j}$ по производным равна нулю, так что они зависят только от $u$. Паре скобок поставим в соответствие характеристическое уравнение

$$
\operatorname{det}\left(\pi_{2}^{i j}(u ; p)-\lambda \pi_{1}^{i j}(u ; p)\right)=0 .
$$

Рассмотрим его корни $\lambda^{1}(u ; p), \ldots, \lambda^{n}(u ; p)$ :

$$
\lambda^{i}(u ; p)=\sum_{k \geqslant 0} \lambda_{k}^{i}(u) p^{k},
$$

где

$$
\lambda_{0}^{i}(u)=w^{i}(u), \quad \lambda_{k}^{i}(u)=0 \quad \text { для нечетных } k .
$$

Условия полупростоты и сильной невырожденности означают, что главные члены $w^{1}(u), \ldots, w^{n}(u)$ этих разложений попарно различны и не являются константами. Отсюда выводится, что эти функции могут быть использованы как локальные координаты на $M^{n}$. Положим

$$
c_{i}=\frac{1}{3} \frac{\lambda_{2}^{i}(u)}{\left\langle d w^{i}, d w^{i}\right\rangle_{1}}, \quad i=1, \ldots, n .
$$


Оказывается, что каждая из функций $c_{i}$ зависит только от одной координаты $w^{i}$. Более того, две бигамильтоновы структуры с одним и тем же бездисперсным пределом (9) эквивалентны, если и только если они имеют одинаковые центральные инварианты.

ПримеР 3. Бигамильтонова структура, задающая иерархию уравнения Кортевега-де Фриза (КдФ)

$$
u_{t}+u u_{x}+\frac{\varepsilon^{2}}{12} u_{x x x}=0,
$$

имеет вид

$$
\{u(x), u(y)\}_{2}-\lambda\{u(x), u(y)\}_{1}=(u(x)-\lambda) \delta^{\prime}(x-y)+\frac{1}{2} u_{x} \delta(x-y)+\frac{1}{8} \varepsilon^{2} \delta^{\prime \prime \prime}(x-y) .
$$

Каноническое преобразование

$$
u=v-\frac{\varepsilon^{2}}{12}\left(\log v^{\prime}\right)^{\prime \prime}+\varepsilon^{4}\left(\frac{v^{\mathrm{IV}}}{288{v^{\prime}}^{2}}-\frac{7 v^{\prime \prime} v^{\prime \prime \prime}}{480 v^{\prime 3}}+\frac{v^{\prime \prime 3}}{90 v^{\prime 4}}\right)^{\prime \prime}+O\left(\varepsilon^{6}\right),
$$

рациональное по производным $v^{\prime}=v_{x}, v^{\prime \prime}=v_{x x}$ и т. д., превращает бездисперсную бигамильтонову структуру

$$
\{v(x), v(y)\}_{2}-\lambda\{v(x), v(y)\}_{1}=(v(x)-\lambda) \delta^{\prime}(x-y)+\frac{1}{2} v_{x} \delta(x-y)
$$

в (14). Здесь $w=u$, единственный центральный инвариант равен константе, $c_{1}=1 / 24$.

ПримеР 4. Бигамильтонова структура уравнения Камассы-Холма

$$
u_{t}-\varepsilon^{2} u_{t x x}=\frac{3}{2} u u_{x}-\varepsilon^{2}\left[u_{x} u_{x x}+\frac{1}{2} u u_{x x x}\right]
$$

задается формулой

$$
\{u(x), u(y)\}_{2}-\lambda\{u(x), u(y)\}_{1}=(u(x)-\lambda) \delta^{\prime}(x-y)+\frac{1}{2} u_{x} \delta(x-y)+\lambda \frac{\varepsilon^{2}}{8} \delta^{\prime \prime \prime}(x-y) .
$$

Бездисперсные пределы для (14) и (18) совпадают. Однако центральный инвариант структуры (18) равен

$$
c_{1}=\frac{1}{24} w, \quad w=u .
$$

Таким образом, иерархии КдФ и Камассы-Холма не эквивалентны.

Теория центральных инвариантов описывает строение пространства инфинитезимальных деформаций бигамильтоновых структур гидродинамического типа. Остается открытой проблема зануления высших препятствий, т. е. проблема существования бигамильтоновой структуры с данным бездисперсным пределом и данными центральными инвариантами. Мы обратимся теперь к специальному подклассу так называемых интегрируемых иерархий топологического типа, связанных с полупростыми фробениусовыми многообразиями. 
Фробениусовы многообразия $M^{n}$ отвечают специальному классу пуассоновских пучков гидродинамического типа. Характеристическим свойством фробениусовых многообразий служит наличие коммутативного и ассоциативного умножения на касательном пучке

$$
\frac{\partial}{\partial u^{i}} \cdot \frac{\partial}{\partial u^{j}}=c_{i j}^{k}(u) \frac{\partial}{\partial u^{k}},
$$

а также метрики нулевой кривизны, задаваемой в плоских координатах невырожденной постоянной симметрической матрицей

$$
\left\langle\frac{\partial}{\partial u^{i}}, \frac{\partial}{\partial u^{j}}\right\rangle=\eta_{i j} .
$$

Требуется наличие локального потенциала $F(u)$ такого, что

$$
\left\langle\frac{\partial}{\partial u^{i}} \cdot \frac{\partial}{\partial u^{j}}, \frac{\partial}{\partial u^{k}}\right\rangle=\frac{\partial^{3} F(u)}{\partial u^{i} \partial u^{j} \partial u^{k}} .
$$

Также предполагается наличие плоского единичного векторного поля $e$ и линейного эйлерова векторного поля $E$ такого, что

$$
[e, E]=e, \quad E F=(3-d) F+\text { квадратичные члены. }
$$

Здесь $d$ - некоторая константа.

Замечательным (и, в известной степени, характеристическим) свойством фробениусовых многообразий является наличие плоского пучка метрик

$$
\begin{aligned}
& \left(d u^{i}, d u^{j}\right)_{1}=\eta^{i j}, \\
& \left(d u^{i}, d u^{j}\right)_{2}=\mathrm{i}_{E} d u^{i} \cdot d u^{j} .
\end{aligned}
$$

Тем самым на пространстве петель фробениусова многообразия $\mathscr{L}\left(M^{n}\right)$ возникает бигамильтонова структура гидродинамического типа и, значит, интегрируемая иерархия. Мы не будем вдаваться в подробности процедуры построения этой иерархии. Укажем лишь одно из ее уравнений:

$$
\mathbf{u}_{t}+\mathbf{u} \cdot \mathbf{u}_{x}=0, \quad \mathbf{u}=\left(u^{1}, \ldots, u^{n}\right) \in M^{n} \simeq T_{\mathbf{u}} M^{n} .
$$

В этой формуле фробениусово многообразие локально отождествляется со своим касательным пространством ввиду наличия плоской метрики.

Фробениусовы многообразия обладают и целым рядом других замечательных свойств. Так, полупростые фробениусовы многообразия (т. е. алгебра в касательном пространстве $T_{u} M^{n}$ полупроста для точек общего положения $u \in M^{n}$ ) описываются через изомонодромные деформации некоторых специальных линейных дифференциальных операторов с рациональными коэффициентами. Примечательной является связь полупростых фробениусовых многообразий с теорией групп, порожденных отражениями. Для наших целей особенно важным является наличие тау-функиий у интегрируемых иерархий, связанных с фробениусовыми многообразиями. Это свойство служит главной мотивировкой для рассмотрения того специального класса интегрируемых иерархий, к которому мы теперь и переходим. 
Основным вопросом здесь является проблема реконструкиии: для каких фробениусовых многообразий система (20) получается как бездисперсный предел некоторой интегрируемой иерархии на $\mathscr{L}\left(M^{n}\right) \otimes \mathbb{R}[[\varepsilon]]$ ? Если такое интегрируемое $\varepsilon$-продолжение существует, то как их перечислить?

Сформулированная выше теорема 2 говорит, что каждая такая иерархия однозначно задается своим бездисперсным пределом и центральными инвариантами. Характеристическим свойством иерархий топологического типа служит то, что

- бездисперсная бигамильтонова структура задается пучком плоских метрик (19), ассоциированным с некоторым полупростым фробениусовым многообразием;

- все центральные инварианты постоянны и равны друг другу.

ТЕОРемА 5. Для любого полупростого фробениусова многообразия существует единственная интегрируемая иерархия топологического типа, связанная с этим многообразием, с иентральными инвариантами

$$
c_{1}=c_{2}=\cdots=c_{n}=\frac{1}{24} \text {. }
$$

Ключом к доказательству этой теоремы служит инвариантность интегрируемых иерархий топологического типа по отношению к вирасоровским симметриям, действующим линейно на тау-функцию.

Обратимся, наконец, к изучению свойств решений построенных уравнений. Естественно спросить, как свойства решений зависят от выбора фробениусова многообразия? Как они меняются с изменением порядка обрывания по $\varepsilon$ ? Какая часть из этих свойств остается справедливой также и для неинтегрируемых возмущений?

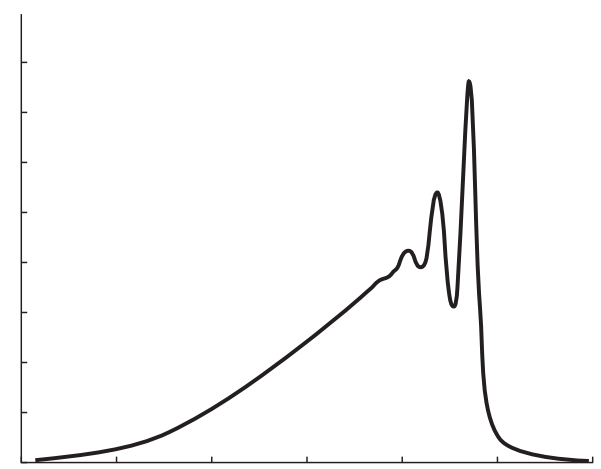

Рис. 1. Критическое поведение решений уравнения КдФ

При малых временах вклад высших $\varepsilon$-поправок мал. Решения бездисперсной системы и ее возмущения начинают различаться вблизи точки градиентной

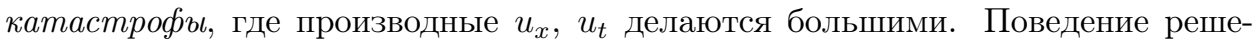
ний гамильтоновых эволюционных систем оказывается качественно отличным от того, что происходит с решениями систем с диссипацией: вместо ударных волн возникают осцилляции с периодом $\sim \varepsilon$ (см. рис. 1$)$. 
ТАБлицА 1. Список примеров фробениусовых многообразий и соответствующих интегрируемых иерархий топологического типа

\begin{tabular}{|c|c|c|}
\hline$n=1$ & $F=\frac{1}{6} v^{3}$ & Кд $\Phi^{1}$ \\
\hline$n=2$ & $F=\frac{1}{2} u v^{2}+u^{4}$ & Буссинеск \\
\hline$n=2$ & $F=\frac{1}{2} u v^{2}+e^{u}$ & Тода \\
\hline$n=2$ & $F=\frac{1}{2} u v^{2}+\frac{1}{2} u^{2}\left(\log u-\frac{3}{2}\right)$ & НУШ \\
\hline$n=2$ & $F=\frac{1}{2} u v^{2}-\mathrm{Li}_{3}\left(e^{-u}\right)$ & Абловиц-Ладик \\
\hline$n=3$ & $\begin{aligned} F= & \frac{1}{2}\left(u w^{2}+u^{2} v\right) \\
& +\frac{1}{6} v^{2} w^{2}+\frac{1}{60} w^{5}\end{aligned}$ & $\begin{array}{l}\text { Иерархия Дринфельда-Соколова } \\
\text { типа } A_{3}, \text { теория пересечений на } \\
\text { пространствах модулей кривых "спина } 3 \text { " }\end{array}$ \\
\hline$n=3$ & $\begin{aligned} F= & \frac{1}{2}\left(u v^{2}+v w^{2}\right) \\
& -\frac{1}{24} w^{4}+4 w e^{u}\end{aligned}$ & $\begin{array}{l}\text { Обобщенная цепочка Тоды для разност- } \\
\text { ного оператора Лакса бистепени }(2,1) ; \\
\text { орбифолдные инварианты Громова- } \\
\text { Виттена для кривой с особой точкой } \\
\text { второго порядка }\end{array}$ \\
\hline$n=3$ & $\begin{aligned} F= & \frac{1}{2}\left(\tau v^{2}+v u^{2}\right) \\
& -\frac{i \pi}{48} u^{4} E_{2}(\tau)\end{aligned}$ & $\begin{array}{l}\text { высшие поправки к эллиптическим } \\
\text { уиземовским асимптотикам, случай КдФ }\end{array}$ \\
\hline$n=4$ & $\begin{aligned} F= & \frac{i}{4 \pi} \tau v^{2}-2 u v w \\
& +u^{2} \log \left[\frac{\pi}{u} \frac{\theta_{1}^{\prime}(0 \mid \tau)}{\theta_{1}(2 w \mid \tau)}\right]\end{aligned}$ & $\begin{array}{l}\text { высшие поправки к эллиптическим } \\
\text { уиземовским асимптотикам, случай } \\
\text { НУШ/Тода }\end{array}$ \\
\hline
\end{tabular}

На первый взгляд поведение решений различных уравнений оказывается совершенно различным. Так, не видно сходства в характере критического поведения, показанном на рис. 1 (случай КдФ) и рис. 2 (случай НУШ).

Тем не менее, есть все основания ожидать, что в действительности в решениях общего положения рассматриваемых гамильтоновых систем встречается лишь конечное число типов критического поведения. В этом и заключается гипотеза универсальности критического поведения, к обсуждению которой мы переходим.

Как видно из табл. 2 , при $n=1,2$ типы критического поведения решений общего вида для невозмущенных систем описываются алгебраическими функциями, известными из теории особенностей (бифуркационная диаграмма особенности типа $A_{3}$, особенность Уитни типа сборки, а также эллиптическая омбилическая особенность Тома). Для возмущенных систем эти особенности заменяются некоторыми специальными решениями уравнений типа Пенлеве и их обобщений. Дадим описание этих решений.

\footnotetext{
13десь под КдФ, Буссинеск, Тода, НУШ и т. д. понимаются иерархии интегрируемых уравнений, связанные соответственно с уравнением Кортевега-де Фриза, уравнением Буссинеска, уравнениями цепочки Тоды, нелинейным уравнением Шрёдингера.
} 


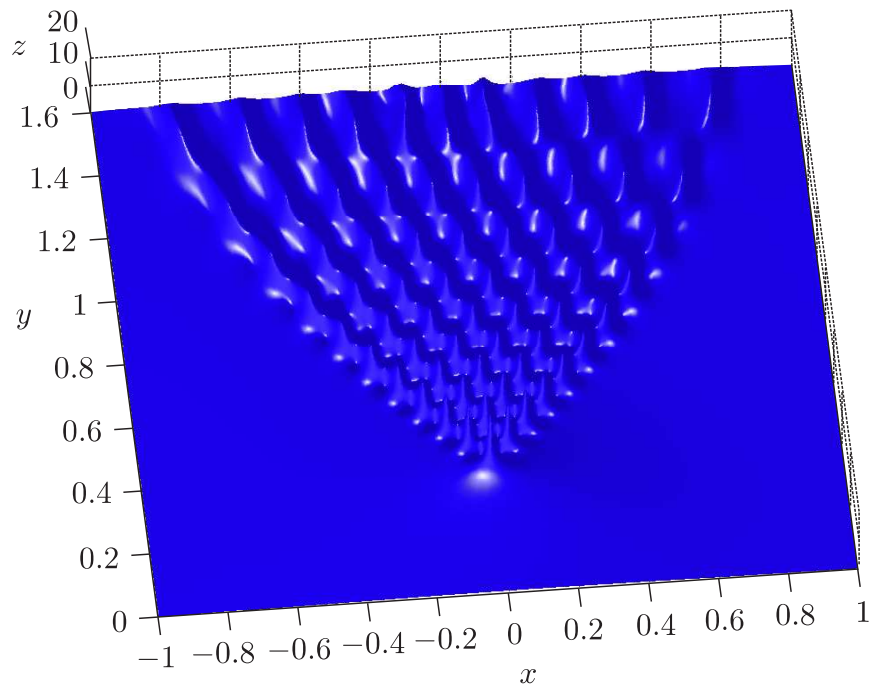

Рис. 2. Критическое поведение решений фокусирующего нелинейного уравнения Шрёдингера $i \psi_{t}+\frac{1}{2} \psi_{x x}+|\psi|^{2} \psi=0$; показан график величины $u=|\psi|^{2}$

ТАБлицА 2. Типы критического поведения решений систем низкого порядка

\begin{tabular}{|c|c|c|}
\hline $\begin{array}{c}\text { Число } \\
\text { зависимых } \\
\text { переменных }\end{array}$ & Бездисперсная система & Возмущенная система \\
\hline$n=1$ & $\begin{array}{l}\text { при } t<0 \text { решение } \\
\text { уравнения } x=u t-\frac{1}{6} u^{3}\end{array}$ & $\begin{array}{l}\text { специальное решение } \\
U(X, T) \text { уравнения } \mathrm{P}_{\mathrm{I}}^{2} \\
\begin{aligned} X & =U T-\frac{1}{6} U^{3} \\
& -\left[\frac{1}{24}\left(U^{\prime 2}+2 U U^{\prime \prime}\right)+\frac{U^{\mathrm{IV}}}{240}\right]\end{aligned}\end{array}$ \\
\hline $\begin{array}{c}n=2 \\
\text { гиперболический } \\
\text { случай }\end{array}$ & $\begin{array}{l}\text { при } t<0 \text { решение системы } \\
\text { в характеристических } \\
\text { переменных } \\
\begin{aligned} x_{+}=r_{+} \\
x_{-}=r_{+} r_{-}-\frac{1}{6} r_{-}^{3}\end{aligned}\end{array}$ & $\begin{array}{l}\text { та же функция } U(X, T), \\
r_{+}=x_{+}+U^{\prime \prime}\left(x_{-}, x_{+}\right), \\
r_{-}=U\left(x_{-}, x_{+}\right)\end{array}$ \\
\hline $\begin{array}{c}n=2 \\
\text { эллиптический } \\
\text { случай }\end{array}$ & $\begin{array}{l}\text { при } z \neq 0 \text { решение } \\
\text { комплексного квадратного } \\
\text { уравнения } z=\frac{1}{2} w^{2}\end{array}$ & $\begin{array}{l}\text { tritronquée (трижды } \\
\text { усеченное) решение } W_{0}(Z) \\
\text { уравнения } \mathrm{P}_{\mathrm{I}} W^{\prime \prime}=6 W^{2}-Z\end{array}$ \\
\hline
\end{tabular}

Начнем с уравнения

$$
X=T U-\left[\frac{1}{6} U^{3}+\frac{1}{24}\left(U^{\prime 2}+2 U U^{\prime \prime}\right)+\frac{1}{240} U^{\mathrm{IV}}\right] .
$$


Это - обыкновенное дифференциальное уравнение для функции $U=U(X)$, зависящее от параметра $T$. В теории уравнений Пенлеве это уравнение обычно рассматривается как высший аналог уравнения Пенлеве-1 (см. ниже). Известно, что при всех значениях параметра $T$ любое решение этого уравнения - мероморфная функция комплексной переменной $X$. Интересующее нас решение, существование которого было доказано лишь в 2006 г. Т. Клаесом и М. Ванлессеном, не имеет полюсов на всей вещественной оси $X$ при вещественных значениях параметра $T$. Это решение, определенное однозначно при всех $(X, T) \in \mathbb{R}^{2}$, мы будем обозначать через $U(X, T)$ (см. рис. 3$)$.
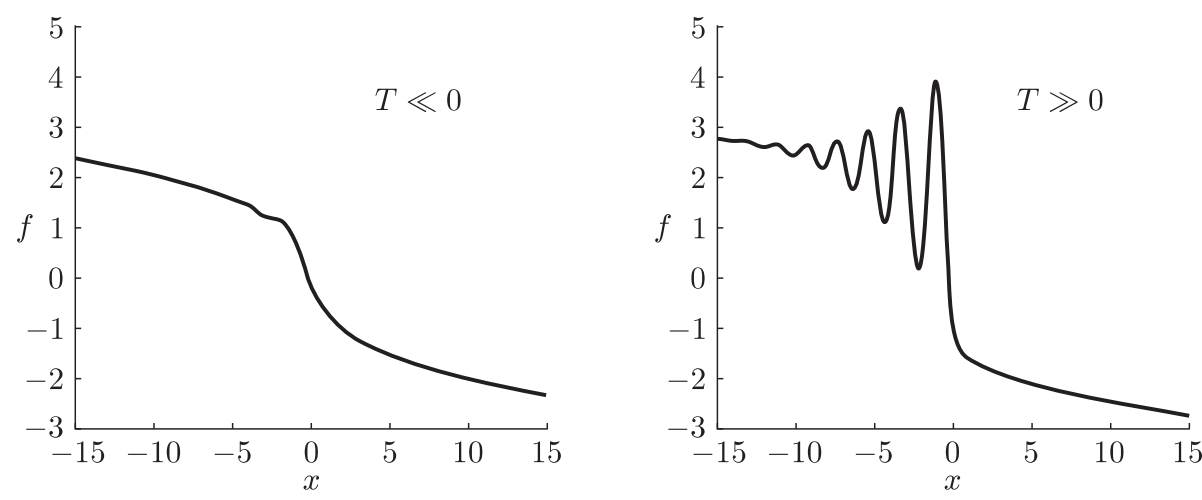

Рис. 3. Решение $U(X, T)$ уравнения (21) для двух значений параметра $T$

Мы готовы к тому, чтобы сформулировать гипотезу универсальности для скалярных гамильтоновых уравнений.

ГиПотезА 6. Рассмотрим общее гамильтоново возмущение уравнения

$$
v_{t}+a(v) v_{x}=0, \quad a^{\prime}(v) \neq 0 .
$$

В окрестности критической точки $\left(x_{0}, t_{0}, v_{0}\right)$ решение общего положения представляется в следующем виде:

$$
u \simeq v_{0}+\left(\frac{\varepsilon^{2} c_{0}}{\kappa^{2}}\right)^{1 / 7} U\left(\frac{x-a_{0}\left(t-t_{0}\right)-x_{0}}{\left(\kappa c_{0}^{3} \varepsilon^{6}\right)^{1 / 7}}, \frac{a_{0}^{\prime}\left(t-t_{0}\right)}{\left(\kappa^{3} c_{0}^{2} \varepsilon^{4}\right)^{1 / 7}}\right)+O\left(\varepsilon^{4 / 7}\right),
$$

где $a_{0}=a\left(v_{0}\right), a_{0}^{\prime}=a^{\prime}\left(v_{0}\right)$, a $c_{0} u \kappa$-некоторые постоянные, решение $U(X, T)$ уравнения (21) описано выше.

Доказательство этой гипотезы для случая решений уравнения КдФ с быстроубывающими аналитическими начальными данными было получено в самое последнее время Т. Клаесом и Т. Гравой.

Как видно из табл. 2, та же самая специальная функция описывает и критическое поведение общих решений гиперболической системы второго порядка. Для случая возмущений эллиптической системы (например, для фокусирующего нелинейного уравнения Шрёдингера) нужна другая специальная функция, к описанию которой мы и переходим.

Речь пойдет о классическом уравнении Пенлеве-1 $\left(\mathrm{P}_{\mathrm{I}}\right)$

$$
W^{\prime \prime}=6 W^{2}-Z \text {. }
$$


Как и выше, все решения этого уравнения - мероморфные функции комплексного переменного $Z$. Асимптотическое расположение полюсов этих функций было исследовано П. Бутру еще в 1913 г. Бутру показал, что линии полюсов общего решения уравнения $\mathrm{P}_{\mathrm{I}}$ накапливаются вдоль пяти лучей

$$
\arg Z=\frac{2 \pi n}{5}, \quad n=0, \pm 1, \pm 2 .
$$

Основным открытием Бутру является доказательство существования специальных решений, для которых эти линии полюсов обрываются вдоль трех последовательных лучей из числа (25). Эти решения, названные Бутру tritronquée, определены однозначно для каждой тройки последовательных лучей.

Рассмотрим, в частности, решение tritronquée, отвечающее тройке лучей вида $(25)$ с $n=0, \pm 1$. Это решение, обозначаемое через $W_{0}(Z)$, по определению имеет лишь конечное число полюсов в секторе $|\arg Z|<4 \pi / 5-\delta$ при любом положительном $\delta$. Нижеследующее утверждение, сформулированное в виде гипотезы Т. Гравой, К. Клайном и автором, утверждает, что этих полюсов нет вовсе.

ГипотезА 7. Решение tritronquе́е $W_{0}(Z)$ является аналитической функцией при всех

$$
|\arg Z|<\frac{4 \pi}{5}
$$

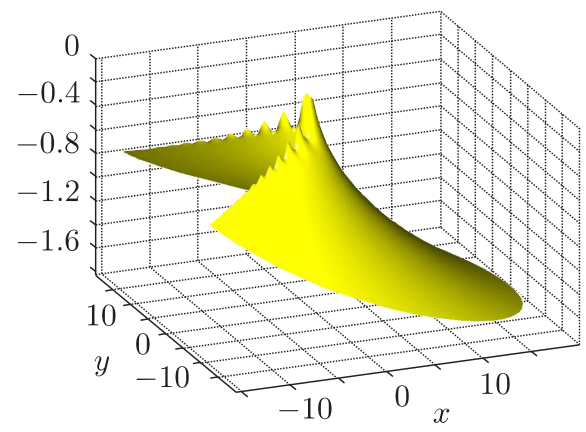

$\operatorname{Re} W_{0}(z)$

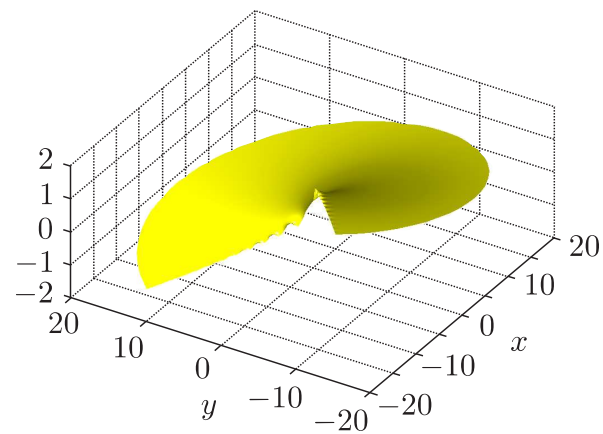

$\operatorname{Im} W_{0}(z)$

Рис. 4. График вещественной (слева) и мнимой (справа) частей решения tritronquée $W_{0}(Z)$ уравнения $\mathrm{P}_{\mathrm{I}}$ в секторе $|\arg Z|<4 \pi / 5$

Из графика, показанного на рис. 4, видно, что полюсов у интересующего нас решения в секторе (26) нет.

Мы готовы к тому, чтобы сформулировать гипотезу универсальности, описывающую критическое поведение гамильтоновых возмущений систем эллиптического типа. Для таких систем имеется пара комплексно сопряженных римановых инвариантов $w$ и $\bar{w}$. Характеристические направления также комплексно сопряжены; обозначим их через $z$ и $\bar{z}$. Как видно из табл. 2, критические точки невозмущенной системы изолированы; в окрестности этих точек решение имеет особенность типа квадратного корня из комплексной величины. 
ГиПотезА 8. Решение общего положения общего гамильтонова возмущения произвольной квазилинейной системы второго порядка эллиптического типа представляется в виде

$$
w \simeq w^{0}+\alpha \varepsilon^{2 / 5} W_{0}\left(\varepsilon^{-4 / 5} z\right)+O\left(\varepsilon^{4 / 5}\right), \quad z=\beta_{+} x+\beta_{-} t+z_{0},
$$

где $\alpha \neq 0, \beta_{ \pm}, z_{0}-$ комплексные константы, причем

$$
|\arg z|<\frac{4 \pi}{5} \quad \text { приммальх }\left|t-t_{0}\right|
$$

при всех $x \in \mathbb{R}$.

Эта гипотеза, впервые сформулированная Т. Гравой, К. Клайном и автором при изучении критического поведения решений фокусирующего нелинейного уравнения Шрёдингера, на сегодняшний день остается не доказанной.

\section{Список литературы}

\section{Часть 1: проблемы классификации}

[1] B. Dubrovin, Y. Zhang, Normal forms of hierarchies of integrable PDEs, Frobenius manifolds and Gromov-Witten invariants, arxiv: math/0108160, 2001.

[2] Si-Qi Liu, Y. Zhang, "Deformations of semisimple bihamiltonian structures of hydrodynamic type", J. Geom. Phys., 54:4 (2005), 427-453.

[3] B. Dubrovin, Si-Qi Liu, Y. Zhang, "On Hamiltonian perturbations of hyperbolic systems of conservation laws. I: Quasitriviality of bi-Hamiltonian perturbations", Comm. Pure Appl. Math., 59:4 (2006), 559-615.

[4] B. Dubrovin, Si-Qi Liu, Y. Zhang, "Frobenius manifolds and central invariants for the Drinfeld-Sokolov bihamiltonian structures", Adv. Math., 219:3 (2008), 780-837.

[5] B. Dubrovin, "On universality of critical behaviour in Hamiltonian PDEs", Geometry, topology, and mathematical physics. S. P. Novikov's seminar: 2006-2007 (Moscow, Russia, 2006-2007), Adv. Math. Sci., 61, Amer. Math. Soc. Transl. Ser. 2, 224, Providence, RI, 2008, 59-109.

\section{Часть 2: универсальность критического поведения}

[6] B. Dubrovin, "On Hamiltonian perturbations of hyperbolic systems of conservation laws. II: Universality of critical behaviour", Comm. Math. Phys., 267 (2006), 117-139.

[7] T. Claeys, M. Vanlessen, "The existence of a real pole-free solution of the fourth order analogue of the Painlevé I equation", Nonlinearity, 20:5 (2007), 1163-1184.

[8] T. Claeys, M. Vanlessen, "Universality of a double scaling limit near singular edge points in random matrix models", Comm. Math. Phys., 273:2 (2007), 499-532.

[9] T. Grava, Ch. Klein, "Numerical solution of the small dispersion limit of Korteweg-de Vries and Whitham equations", Comm. Pure Appl. Math., 60:11 (2007), 1623-1664.

[10] T. Claeys, T. Grava, Universality of the break-up profile for the KdV equation in the small dispersion limit using the Riemann-Hilbert approach, arxiv: math/0801.2326, 2008.

[11] B. Dubrovin, T. Grava, C. Klein, "On universality of critical behaviour in the focusing nonlinear Schrödinger equation, elliptic umbilic catastrophe and the tritronquée solution to the Painlevé-I equation", J. Nonlinear Sci. (to appear); arxiv: math/ $0704.0501,2007$.

\section{Б. А. Дубровин (B. A. Dubrovin)}

Математический институт им. В. А. Стеклова РАН;

Поступила в редакцию International School for Advanced Studies (SISSA)

E-mail: dubrovin@sissa.it 